\title{
AN INTEGRAL EQUATION APPROACH TO BOUNDARY VALUE PROBLEMS OF CLASSICAL ELASTOSTATICS
}

\author{
BY \\ FRANK J. RIZZO \\ University of Washington
}

Summary. The analogy between potential theory and classical elasticity suggests an extension of the powerful method of integral equations to the boundary value problems of elasticity. A vector boundary formula relating the boundary values of displacement and traction for the general equilibrated stress state is derived. The vector formula itself is shown to generate integral equations for the solution of the traction, displacement, and mixed boundary value problems of plane elasticity. However, an outstanding conceptual advantage of the formulation is that it is not restricted to two dimensions. This distinguishes it from the methods of Muskhelishvili and most other familiar integral equation methods. The presented approach is a real variable one and is applicable, without inherent restriction, to multiply connected domains. More precisely, no difficulty of the order of determining a mapping function is present and unwanted Volterra type dislocation solutions are eliminated a priori. An indication of techniques necessary to effect numerical solution of the resulting integral equations is presented with numerical data from a set of test problems.

1. Introduction. The strong analogy between potential theory and classical elasticity theory has been exploited most notably by Betti [1], Somigliana [2], Lauricella [3], and other geometers of the Italian school. In particular, Betti's generil method of integrating the equations of elasticity may be regarded as a direct exte. ion of the potential theoretic methods of Green and Poisson. Further, the powerfu! theory of singular integral equations has been extended by Fredholm [4] and Lauricella [3] to attack elasticity problems. This latter approach circumvents, somewhat, the inherent difficulty with Betti's method, that of constructing the tensor counterpart of a Green's function. More recently Sherman [5], Mikhlin [6], and Muskhelishvili [7] have used singular integral equations in treating plane elasticity problems via the elegant complex function approach.

Rather effective treatment can be given, however, to two-dimensional elasticity problems by real variable methods using integral equations. This is indicated in the paper by Jaswon [8]. His approach is free from the necessity of finding influence functions and mapping functions for the regions in question, often a formidable task even for simply connected domains. On the other hand, it inherently applies only to two dimensions, and works with a stress function irstead of directly with the assigned boundary quantities. A real variable formulation of problems in terms of the assigned boundary values only is possible, fortunatrly, and is the type of formulation presented in this paper.

The presented approach depends on the knowledge of the singular solution to the Navier-Cauchy elasticity equations in two dimensions which corresponds to a concentrated force. This singular solution gives rise to a vector identity similar to Green's

Received November 16, 1965; revised manuscript received August 15, 1966. 
third identity for Laplace's equation. Taking the field point to lie on the boundary contour, a boundary formula is obtained which is a relation between boundary displacements and corresponding boundary tractions. Since either of these boundary quantities, in principle, determines the other, the formula provides a constraint bctween them, which, as shown apparently for the first time in this paper, generates a set of simultaneous integral equations from suitable boundary data. These are of different types according as data appropriate to the first, second, or mixed boundary value problems are prescribed. The unknowns in the equations are boundary tractions or displacements directly. Once these are obtained, the displacement field is generated by means of the vector identity. It may be remarked that analogous, though simpler, types of integral equations generated by Green's third identity on the boundary (Green's boundary formula), have been successfully solved numerically by Jaswon and Ponter [9] and by Symm [10].

Problems for multiply connected regions may be considered without fundamental restriction. No mapping function need be found and tractions on each boundary need not separately be equilibrated. Also, Volterra dislocation solutions are inherently eliminated from the formulation at the outset. This then is a unified direct approach to all three fundamental boundary value problems of elasticity. The solution to each is generated with one and the same formulation from a knowledge of boundary data alone offering generality, practicality, convenience, and economy of effort.

This paper deals entirely with two-dimensional problems and includes numerical data from a trial set of problems to lend confidence to the method. Data from an extensive set of problems representing a more complete exploration and test of the method will be discussed in subsequent papers. An outstanding conceptual advantage of the boundary formula approach is that it applies to three dimensions as well as two with similarly defined integral equations arising in eac' case. The three-dimensional counterpart of the presented approach and several conver ient auxiliary function formulations including one emanating from the Papkovich-Neuber decomposition are currently under investigation.

2. Integral equation formulation. The Navier-Cauchy equations of elasticity in the absence of body forces take the form

$$
(\lambda+\mu) \vartheta_{, i}+\mu \nabla^{2} u_{i}=0, \quad \vartheta=u_{i, i}
$$

and embrace the problems of plane strain and generalized plane stress if all variable quantities are considered functions of planar Cartesian coordinates $x_{i}$. In Eq. (2.1) $u_{i}$ is the displacement vector, $\vartheta$ the dilatation, and the remaining symbols have their usual significance.

Let the body under consideration first be finite and bounded by a single smooth contour $C$ which, as described by Muskhelishvili [7], admits a representation in the form $x_{i}=x_{i}(s)$. The first derivatives $x_{i}^{\prime}(s)$, are continuous and never simnltaneously zero. The paraneter $s$ is arc length along the contour from some arbitrary origin on it. The region uccupied by the body is traversed in the positive sense keeping the region on the left. The positive tangent at a boundary point $Q$ agrees in sense with that of

'The usual indicial notation of Cartesian tensor analysis is used. Latin subscripts have the range $(1,2)$ and summation over repeated subscripts is implied. Subscripts preceded by a comma indicate differentiation with respect to the corresponding Cartesian coordinate. Kronecker's delta and the alternating symbol are denoted by $\delta_{i j}$ and $e_{i j k}$, respectively. 
positive traverse and the positive unit normal $n_{i}$ at $Q$ points away from the region. The interior open region of the plane thus defined is labeled $\sigma$ and the exterior open region $\sigma^{\prime}$. Points of $\sigma$ are designated $p$, points of $\sigma^{\prime}$ are designated $p^{\prime}$. Points of $C$ are designated $P$ or $Q$.

For the body $(\sigma+C)$ in equilibrium under the action of boundary tractions $t_{i}$ the relation

$$
\Gamma\left[u_{i}\right]=t_{i}=\lambda \vartheta n_{i}+\mu n_{j}\left(u_{i, i}+u_{i, i}\right)
$$

must hold on $C$ in which $\Gamma$ is the indicated operator.

Let $x_{i}$ and $\xi_{i}$ be two points of $\sigma$ and define

$$
r=\left[\left(x_{1}-\xi_{1}\right)^{2}+\left(x_{2}-\xi_{2}\right)^{2}\right]^{1 / 2} .
$$

Further, let $U_{i j}$ be the tensor field defined by

$$
U_{i j}=\delta_{i j} \log r+M r_{, i} r_{, i} .
$$

The displacement vectors $u_{i}^{\prime}$ defined by

$$
u_{i}^{\prime}=U_{i j} e_{i}
$$

in which $e_{i}$ are unit base vectors both satisfy Eqs. (2.1) in $\sigma$ provided

$$
r \neq 0, \quad M=-\frac{(\lambda+\mu)}{(\lambda+3 \mu)} \text {. }
$$

These displacement vectors arise from concentrated forces of magnitude $-4 \pi \mu(\lambda+2 \mu)$ / $(\lambda+3 \mu)$ acting at the point $p=\xi_{i}$ in the $x_{i}$ directions respectively. These singularities are termed nuclei of strain by Love [11].

The traction vectors $t_{i}^{\prime}$ on $C$ corresponding to $u_{i}^{\prime}$ are computed from I $\gamma .(2.2)$ and may be written

$$
t_{i}^{\prime}=T_{i j} e_{i}
$$

in which

$$
T_{i j}=\frac{\partial}{\partial n_{Q}} \log r\left[k \delta_{i j}-4 \mu M r_{, i} r_{, i}\right]+k\left[(\log r)_{, i} n_{i}-(\log r)_{, i} n_{i}\right] .
$$

In the above expression for the tensor field $T_{i i}$, the derivatives are with respect to the coordinates $x_{i}$ at $Q$ and $r=r(p, Q)$. Also, $k$ has the value $2 \mu^{2} /(\lambda+3 \mu)$.

The vectorial form of Betti's reciprocal work theorem can be written

$$
\int_{C+m}\left(u_{i} T_{i i}-t_{i} U_{i j}\right) d Q=0
$$

where $d Q$ is an element of arc length at $Q$ and in which the point $p=\xi_{i}$ of $\sigma$ has been cxcluded by a small circle $m$ of radius $\delta$ because of the singular nature of $U_{i j}$ and $T_{i i}$ for $r=0$. The vectors and their derivatives are assumed nonsingular and sufficiently continuous for usual validity of the theorem. These vectors correspond to an equilibrated stress state and body forces associated with $U_{i j}, T_{i j}$ are taken as zero with no loss in generality. Now it is easy to show that

$$
\lim _{i \rightarrow 0} \int_{m} t_{i} U_{i j} d Q=0, \quad \lim _{\delta \rightarrow 0} \int_{m} u_{i} T_{i i} d Q=-\alpha^{-1} u_{i}(p)
$$


in which $\alpha=(\lambda+3 \mu) / 4 \pi \mu(\lambda+2 \mu)$. Equation (2.3) yields, therefore, the cxpression

$$
u_{i}(p)=\alpha \int_{c}\left[u_{i}(Q) T_{i i}(p, Q)-t_{i}(Q) U_{i j}(p, Q)\right] d Q
$$

which is the plane counterpart of Somigliana's integral (cf. Love [11, p. 245]).

If the region occupied by the body is $\left(\sigma^{\prime}+C\right)$, some attention to detail must be given in using Betti's theorem. If the tractions are equilibrated on $C,{ }^{2}$ and $u_{i}\left(p^{\prime}\right)=o(1)$ as $p^{\prime} \rightarrow \infty$ uniformly, Betti's theorem is valid as used above and Eq. (2.5) holds for $p$ replaced by $p^{\prime}$.

Equation (2.5) is a relation among a vector $u_{i}(p)$ which satisfies Eq. (2.1), its boundary values $u_{i}(Q)$, and its corresponding boundary tractions $t_{i}(Q)$. This relation is the analogue of Green's third identity of potential theory which expresses a harmonic function in terms of the boundary values of the function and its normal derivative.

The integrals present in Eq. (2.5), i.e.,

$$
\phi_{i}(p)=\int_{c} t_{i}(Q) U_{i j}(p, Q) d Q, \quad \psi_{i}(p)=\int_{c} u_{i}(Q) T_{i j}(p, Q) d Q
$$

are likened to the single and double layer scalar integrals of potential theory. The properties of the integrals as the boundary $C$ is crossed are desired. If

$$
\lim _{p \rightarrow P} \phi_{i}(p)=\phi_{i}(P), \quad \lim _{p \rightarrow P} \psi_{i}(p)=\psi_{i}(P)
$$

then letting $p \rightarrow P$ in the second of these integrals yields

$$
\psi_{i}(P)=\pi(k-2 \mu M) u_{i}(P)+\int u_{i}(Q) T_{i j}(P, Q) d Q .
$$

This result is readily verified provided $u_{i}$ is assume to satisfy a Hölder condition on $C$ and provided the singular integral is understood in the sense of a Cauchy Principal Value. ${ }^{3} \mathrm{~A}$ similar but simpler limiting procedure for the single layer type integral gives

$$
\phi_{i}(P)=\int_{c} t_{i}(Q) U_{i j}(P, Q) d Q .
$$

Since

$$
u_{i}(P)=\alpha\left[\psi_{i}(P)-\phi_{i}(P)\right]
$$

and since

$$
\alpha \pi(k-2 \mu M)=1 / 2
$$

there results

$$
K\left[u_{i}\right]=u_{i}(P)+2 \alpha \int_{c}\left[t_{i}(Q) U_{i j}(P, Q)-u_{i}(Q) T_{i j}(P, Q)\right] d Q=0
$$

in which the operator $K$ will be regular according to the classification of singular oferators by Muskhelishvili [12] for all admissible values of Poisson's ratio. The exprcssion (2.6) is the theory of elasticity analogue of Green's boundary formula.

${ }^{2}$ For exterior or interior multiply connected regions, the meaning of $C$ is clear after suitable cits are introduced in the standard fashion to render such regions simply connected.

${ }^{3}$ Details surrounding the principal value notion and a statement of the Hölder condition can be found in Muskhelishvili [12]. 
Given $t_{i}$ on $C$ (traction problem) the two components of Eq. (2.6) form a pair of integral equations for the unknowns $u_{i}$ on $C$. As might be expected, the rigid body displacement vector

$$
u_{i}(P)=a_{i}+b e_{i j 3} \xi_{i}(P)
$$

satisfies the homogeneous form of these equations, i.e.,

$$
u_{i}(P)-2 \alpha \int_{C} u_{i}(Q) T_{i j}(P, Q) d Q=0
$$

where $a_{i}$ and $b$ are arbitrary constants. Application of the Fredholm alternative yields

$$
\int_{C} t_{i}(Q) d Q=\int_{C} e_{3 i k} x_{j}(Q) t_{k}(Q) d Q=0
$$

as the necessary and sufficient conditions for the solubility of Eq. (2.6). These are, of course, the conditions that the body be in equilibrium.

It should be noted that although the Fredholm alternative was employed above, Eqs. (2.6) are not Fredholm integral equations. The presence of the term

$$
T_{{ }_{i}}^{*}=k\left[(\log r){ }_{i} n_{i}-(\log r),{ }_{i} n_{i}\right]
$$

in the kernel function $T_{i i}$ is responsible for this. More precisely

$$
\lim _{Q \rightarrow P^{P}}|Q-P| T_{i i}^{*}(P, Q) \neq 0
$$

whereas a limit of zero is required for Fredholm kernels. Nevertheless, the index $\kappa$ (cf. Muskhelishvili [12, Chap. 19]) computed from $T_{i j}^{*}$ is zero and the Fredholm alternative is applicable.

To effect a numerical solution of the traction problem the rigid body sortion of the solution must be eliminated. The manner of accomplishing this is consi ,red in the subsequent discussion on numerical procedures.

Given $u_{i}$ on $C$ (displacement problem) the two components of Eq. (2.6) are a pair of integral equations in the unknowns $t_{i}$ on $C$. It is expected that these equations have a unique solution $t_{i}$ on $C$ for arbitrary $u_{i}$ on $C$. This is equivalent to the fact that the homogeneous equations

$$
\int_{C} t_{i}(Q) U_{i j}(P, Q) d Q=0
$$

have no nonzero solutions. This may be proven as follows. Let

$$
u_{i}^{*}(p)=\int_{C} \lambda_{i}(Q) U_{i j}(p, Q) d Q, \quad \int_{C} \lambda_{i}(Q) d Q=0
$$

be a displacement field defined in $\sigma$. The field $u_{i}^{*}\left(p^{\prime}\right)$ is also defined in $\sigma^{\prime}$ and vanishes uniformly as $p^{\prime} \rightarrow \infty$. Further, let

$$
\lim _{p \rightarrow P} t_{i}^{*}(p)=t_{i}^{*}(P)^{+}, \quad \lim _{p^{\prime} \rightarrow P} t_{i}^{*}\left(p^{\prime}\right)=t_{i}^{*}(P)^{-}
$$

in which

$$
\begin{aligned}
& t_{i}^{*}(P)^{+}=k_{i_{1}} \lambda_{j}(P)+\int_{C} \Gamma\left[\lambda_{i}(Q) U_{i j}(P, Q)\right] d Q, \\
& t_{i}^{*}(P)^{-}=-k_{1} \lambda_{j}(P)+\int_{C} \Gamma\left[\lambda_{i}(Q) U_{i j}(P, Q)\right] d Q
\end{aligned}
$$


where $k_{1}$ is a nonzero constant and $\Gamma$ is the vector operator defined by Eq. (2.2). Since $u_{i}^{*}$ is continuous across $C$, if $u_{i}^{*}(P)=0$ the field $u_{i}^{*}=0$ in the entire plane. Thus

$$
t_{i}^{*}(P)^{+}=t_{i}^{*}(P)^{-}=0
$$

and subtracting the second of Eqs. (2.9) from the first yields

$$
\lambda_{i}(P)=0 .
$$

This completes the proof.

With knowledge of $u_{i}=u_{i}^{1}$ on part of $C$ and $t_{i}=t_{i}^{2}$ on the remaining part (mixed problem), a set of four equations in the unknowns $u_{i}^{2}$ and $t_{i}^{1}$ on $C$ is obtained. For this and the two previous boundary value problems the displacement field is generated by means of Eq. (2.5).

Should the region occupied by the body be multiply connected, the methods presented above may be extended without inherent difficulty. Indeed, if boundary data are given on all contours, and the integral in Eq. (2.5) is taken over each of the contours in the proper sense (see footnote 2), Eq. (2.5) yields a single-valued displacement vector in any multiply-connected domain. Also, if the integral in the boundary relation (2.6) is taken over the contours in a similar fashion, the reduction to integral equations of the mentioned types follows immediately.

3. Numerical techniques. Methods of approximating the integral equations which arise in this paper reflect the work by Symm [10] but modifications and additions are included. The singular kernels which arise in Eqs. (2.6) are more complicated than Symm's kernels and particular attention must be given singular kernels whose integrals exist only in the sense of a Principal Value.

Assume first that the displacement problem is to be solved. The contour $C$ is divided into $n$ intervals and a set of $n$ nodal points are : n.ted on $C$. The $\mu$ th nodal point $Q_{\mu}$ is located at the center of the $\mu$ th interval. For purp ines of integrating over each interval assume the unknown tractions $t_{i}(Q)$ remain constant over each interval. Equations (2.6) are then replaced by the linear algebraic system

$$
\sum_{\mu=1}^{n} t_{i \mu}\left(Q_{\mu}\right) \int_{\mu} U_{i j}(P, Q) d Q=\Theta_{i}\left(P_{\nu}\right) \quad(\nu=1,2, \cdots n)
$$

in which $\Theta_{i}\left(P_{v}\right)$ are known functions involving the assigned boundary displacements. The end points of the indicated $\mu$ th interval of integration lie midway between $Q_{\mu-1}$ and $Q_{\mu+1}$ and these end points are denoted by $Q_{\mu-1 / 2}$ and $Q_{\mu+1 / 2}$ respectively.

The solutions $t_{i \mu}$ of the system (3.1) give rise to a displacement field

$$
u_{i}(p)=-\alpha\left[\sum_{\mu=1}^{n} t_{i \mu}\left(Q_{\mu}\right) \int_{\mu} U_{i j}(p, Q) d Q-\Theta_{i}^{\prime}(p)\right]
$$

in which

$$
\Theta_{i}^{\prime}(p)=\int_{C} u_{i}(Q) T_{i j}(p, Q) d Q
$$

is known.

To evaluate the coefficients of $t_{i \mu}$ in Eqs. (3.1), note that Point $P$, will $i m$ de with one of the points $Q_{\mu}$ once in each cquation of the system (3.1). For the remu i. ing possibilities the integrals in (3.1) are well defined and a Simpson approximatio: is used. When $P_{\nu}=Q_{\mu}$, i.c., $P$, within the intcrval of integration, the expression 
$\int_{\mu} \log r d Q=r\left(Q_{\mu}, Q_{\mu-1 / 2}\right)\left[\log r\left(Q_{\mu}, Q_{\mu-1 / 2}\right)-1\right]$

$$
+r\left(Q_{\mu}, Q_{\mu+1 / 2}\right)\left[\log r\left(Q_{\mu}, Q_{\mu+1 / 2}\right)-1\right]
$$

is found by direct integration assuming $d s \doteq d r$ for the appropriate term in Eqs. (3.1). For the remaining integrals in Eqs. (3.1) it is helpful to note that

$$
r_{, 1}=\cos \theta, \quad r_{, 2}=\sin \theta
$$

where $\theta$ is the angle between $r(P, Q)$ and the $x_{1}$ axis. Although $\theta$ is not well defined when $r=0$, the integrals

$$
\int_{\mu} r_{, i} r_{, i} d Q
$$

do exist. But because of this character for $r=0$, a trapezoidal approximation over the interval $Q_{\mu-1 / 2}, Q_{\mu+1 / 2}$ is used for such integrals when $P_{\nu}=Q_{\mu}$.

To solve the traction problem Eqs. (2.6) are replaced by the system

$$
u_{i}\left(P_{\nu}\right)-2 \alpha \sum_{\mu=1}^{n} u_{i \mu}\left(Q_{\mu}\right) \int_{\mu} T_{i j}\left(P_{\nu}, Q\right) d Q=\Omega_{j}\left(P_{\nu}\right)
$$

in which $\Omega_{j}(P$,$) are known functions involving the assigned boundary tractions. To$ ensure a unique solution to the system (3.3) it is necessary to specify the rigid bodymotion. This can be accomplished by suitably prescribing three displacements components of three convenient boundary points as is specifically demonstrated in the next section. Three equations are then eliminated. The solutions $u_{i \mu}$ of this reduced system give rise to a displacement field

$$
u_{i}(p)=\alpha\left[\sum_{\mu=1}^{n} u_{i \mu} \int_{\mu} T_{i j}(p, Q) d Q-\Omega_{j}^{\prime}(p)\right]
$$

in which

$$
\Omega_{i}^{\prime}(p)=\int_{c} t_{i}(Q) U_{i j}(p, Q) d Q
$$

is known.

Regarding the coefficients of $u_{i \mu}$ in Eqs. (3.3); note that since $\left(\partial / \partial n_{\diamond}\right) \log r d Q=d \theta$, and since $r_{, i}$ have the interpretations $\cos \theta, \sin \theta$, integrations may be carried out exactly for $P_{v}$ outside the interval of integration. For $P_{v}$ within the interval of integration, the integrals

$$
\beta_{\mu i j}=\int_{\mu} T_{i j}^{0}\left(Q_{\mu}, Q\right) d Q,
$$

in which $T_{i j}^{0}=T_{i j}-T_{i j}^{*}$, are more conveniently evaluated by the equivalent calculation

$$
\beta_{\mu i i}=\int_{C} T_{i, i}^{6}\left(Q_{\mu}, Q\right) d Q-\int_{C-\mu} T_{i j}^{0}\left(Q_{\mu}, Q\right) d Q .
$$

Using the known relitions

$$
\int_{i} \frac{\partial}{\partial n_{Q}} \log r d Q=\pi, \quad \int_{C} r_{, i} r_{, i} \frac{\partial}{\partial n_{Q}} \log r d Q=\frac{\pi}{2} \delta_{i j}
$$


in which $r=r(P, Q)$, the first integral of (3.5) is easily found (cf. MacMillan [13]). The second integration can be performed directly. The only remaining integrals which require attention, i.e.,

$$
\beta_{\mu i i}^{*}=\int_{\mu} T_{i i}^{*}\left(Q_{\mu}, Q\right) d Q
$$

have the common value zero when evaluated in the required sense of the Principal Value.

For the mixed boundary value problem, the displacements $u_{i}=u_{i}^{1}$ on part $C_{1}$ of $C$ and the tractions $t_{i}=t_{i}^{2}$ on the remaining part are known. The curves $C_{1}$ and $C_{2}$ are divided into $n_{1}$ and $n_{2}$ intervals, respectively. Equations (2.6) are then replaced by the system

$$
\begin{array}{r}
u_{i}\left(P_{\nu}\right)+2 \alpha\left[\sum_{\mu=1}^{n_{1}} t_{i \mu}^{1}\left(Q_{\mu}\right) \int_{\mu} U_{i j}\left(P_{\nu}, Q\right) d Q-\sum_{\mu=1}^{n_{3}} u_{i_{\mu}}^{2}\left(Q_{\mu}\right) \int_{\mu} T_{i j}\left(P_{\nu}, Q\right) d Q\right] \\
=\Omega_{j}^{1}\left(P_{\nu}\right)-\Theta_{j}^{2}\left(P_{\nu}\right)
\end{array}
$$

in which $\Omega_{i}^{1}\left(P_{v}\right)$ and $\Theta_{i}^{2}\left(P_{v}\right)$ are known functions of the prescribed displacements and tractions on $C_{1}$ and $C_{2}$, respectively. Once these equations are solved for the quantities $u_{i}^{2}$ on $C_{2}$ and $t_{i}^{1}$ on $C_{1}$, the displacement field $u_{i}(p)$ is obtained from Eq. (2.5) in which, of course, the integrations are performed in the manner of Eq. (3.2) and Eq. (3.4).

All numerical operations are well suited for computation by a digital computer working upon the input data

$$
Q_{\mu}=x_{i \mu}, \quad Q_{\mu \pm 1 / 2}=x_{i \mu \pm 1 / 2} .
$$

The functions $\Theta_{i}\left(P_{v}\right)$ and $\Omega_{i}\left(P_{v}\right)$ may be entered as data or computed separately by the machine. A single program may be written which 'rms and solves the necessary equations and then generates the desired displacement tidds.

4. Numerical data. The numerical solutions to a number of elementary boundary value problems are obtained as a check on the validity of the approximations outlined in Sec. 3. Computed data are thus compared with easily obtained analytical solutions. The domains considered are the circle, the circular ring, the ellipse, and the rectangle. The rectangular domain is regarded as a problem in plane stress; all others in plane strain. For generality and ease in programming, all functions $\Theta_{i}$ and $\Omega_{i}$ are computed by the machine assuming known tractions and displacements constant over the $\mu$ th interval. Also, no advantage of obvious symmetry is taken in programming. All quantities associated with each nodal point are calculated individually.

For each domain a right-handed coordinate system $x_{i}$ is used; the origin of coordinates is coincident with the centroid of the domain and the coordinate axes for the ellipse and rectangle coincide with the major and minor axes for these shapes.

A traction problem for the circular domain is considered first. Twelve icdal points on the bomilary are located and numbered for identification as the hour rositions on the clork. Node three is thus on the $x_{1}$ axis. A tensile traction $N=P_{0} \sin ^{2} \phi$ is applied to the boundary; $P_{0}$ is constant ancl $\phi$ is the angle of a radius of the circle with the positive $x_{1}$ axis. The rigid body motion is effectively reduced to zero and th: : sociated boundary displacement components are calculated. Next, as a first check on the results, a tensile traction $N=P_{0} \cos ^{2} \phi$ is applied and the net boundary displace rent components are compared with those expected from the stress $N=P_{0}$ acting alone. A 
second run on this problem is obtained with twenty-four nodal points by adding a node midway between each of the original twelve. Again node one is in the same position and the remaining nodes are numbered in sequence according to a clockwise traverse. An improvement in the accuracy of calculated data is observed as expected. The expected symmetry in boundary data is obtained, hence, data for the first quadrant only are given in Table I.

To specify the rigid body motion for these and subsequent traction problems, the $u_{2}$ components of displacement for both nodal points on the $x_{1}$ axis and the $u_{1}$ component for the point on the $x_{2}$ axis are taken as zero. Since $u_{i \mu}$ at the nodes really represent displacement components of all material points on the associated boundary intervals, this amounts, in principle, to an overspecification of boundary data. But because of the character of the actual relative displacement over the intervals involved and the relatively small arc lengths even for twelve segments, serious error is not introduced.

A displacement problem for the circular ring is considered by assuming the inner boundary fixed and the outer boundary rotated a constant amount $\phi_{0}$ clockwise. The nodal pattern for the inner boundary is exactly that for the solid circle for twelve nodes. Twelve more nodes are located on the outer boundary occupying the half-hour positions on the clock. To continue the numbering, node thirteen occupies the half-past twelve position. The remaining are numbered in sequence clockwise giving a total of twentyfour. The tractions $t_{i}^{1}$ and $t_{i}^{2}$ on the inner and outer boundaries respectively, corresponding to the given boundary displacement, are calculated and compared with the expected analytical values. Next, to pose a mixed problem, $t_{i}^{2}$ is applied keeping the inner bound-

TABLE I. Boundary data for circular domain, traction $N=P_{0} \sin ^{2} \phi$

Radius $\rho=10^{4}$ in., Poisson's ratio $\nu=.25$

Young's Modulus $E=10^{7}$ p.s.i., $P_{0}=1600$ p.s.i.

Boundary $u_{i_{\mu}}$ (in.), $n=12$

Numerical

Analytical

\begin{tabular}{c|c|c|c|c}
\hline Node & $u_{1 \mu}$ & $u_{2 \mu}$ & $u_{1 \mu}$ & $u_{2 \mu}$ \\
\hline 12 & 0 & 1.2690 & 0 & 1.3333 \\
1 & 0.2531 & 0.8689 & 0.3333 & 0.8660 \\
2 & -0.0219 & 0.2360 & 0.0001 & 0.1667 \\
3 & -0.2910 & 0 & -0.3333 & 0 \\
\hline
\end{tabular}

Numerical

Boundary $u_{i \mu}$ (in.), $n=24$

Analytical

\begin{tabular}{c|c|c|c|c}
\hline Node & $u_{1 \mu}$ & $u_{2_{\mu}}$ & $u_{1 \mu}$ & $u_{2 \mu}$ \\
\hline 23 & 0 & 1.2984 & 0 & 1.3333 \\
24 & 0.2075 & 1.1769 & 0.2357 & 1.2016 \\
1 & 0.2916 & 0.8660 & 0.3333 & 0.8660 \\
2 & 0.2013 & 0.4960 & 0.2357 & 0.4714 \\
3 & -0.0120 & 0.2015 & 0.0001 & 0.1667 \\
4 & -0.2244 & 0.0477 & -0.2357 & 0.0231 \\
5 & -0.3123 & 0 & -0.3333 & 0
\end{tabular}


ary fixed. Values $t_{i}^{1}$ and $u_{i}^{2}$ on the inner and outer circles are then calculated. Boundary data for the first quadrant are given in Table II.

The boundary of the ellipse is divided into twenty-four equal segments. Node one is on the positive $x_{1}$ axis and the remaining nodes are numbered in sequence according to a clockwise traverse. The coordinates of the nodal points for equal spacing are entered as data from computations by a special subroutine which uses the equation of the ellipse. A uniform tensile traction $N=P_{0}$ is applied normal to the boundary and the corresponding boundary displacement components are calculated. First quadrant boundary data are given in Table III.

The boundary of the rectangle is divided into twenty-eight segments by dividing the long and short sides into nine and five segments, respectively. Nodal points coincident with corners where the contour has no unique tangent are thus avoided. Node one is on the positive $x_{1}$ axis again and the remaining nodes are numbered as for the ellipse.

TABLE II. Boundary data for circular ring

Radii: $\rho_{1}=10^{4}$ in., $\rho_{2}=2 \times 10^{4}$ in.; Poisson's ratio $\nu=.25$

Young's Modulus $E=10^{7}$ p.s.i.

Displacement prob., $\phi_{0}=5 \times 10^{-5}$ radian

Tractions $t_{i \mu}$ (p.s.i.)

Numerical

Analytical

\begin{tabular}{c|c|c|c|c}
\hline Node & $t_{1 \mu}$ & $t_{2 \mu}$ & $t_{1 \mu}$ & $t_{2 \mu}$ \\
\hline 12 & -543.2 & 0 & -532.0 & 0 \\
1 & -470.4 & 271.6 & -461.5 & 266.0 \\
2 & -271.6 & 470.4 & -266.0 & 461.5 \\
3 & 0 & 543.2 & 0 & 532.0 \\
13 & 140.4 & -37.6 & 128.7 & -34.5 \\
14 & 102.7 & -102.7 & 94.3 & -94.3 \\
15 & 37.6 & -140.4 & 34.5 & -128.7 \\
\hline
\end{tabular}

Mixed prob., uniform shear 532 p.s.i. on $C_{2}$

Tractions $t_{i \mu}$ (p.s.i.)

Numerical

Analytical

\begin{tabular}{c|c|c|c|c} 
Node & $t_{1 \mu}$ & $t_{2 \mu}$ & $t_{1 \mu}$ & $t_{2 \mu}$ \\
\hline 12 & -497.2 & 0 & -532.0 & 0 \\
1 & -430.6 & 248.6 & -461.5 & 266.0 \\
2 & -248.6 & 430.6 & -266.0 & 461.5 \\
3 & 0 & 497.2 & 0 & 532.0
\end{tabular}

Displacements $u_{i \mu}$ (in.)

\begin{tabular}{cc|c|c|c} 
& $u_{1 \mu}$ & $u_{2 \mu}$ & $u_{1 \mu}$ & $u_{2_{\mu}}$ \\
\hline 13 & 0.8841 & -0.2369 & 0.9659 & -0.2588 \\
14 & 0.6472 & -0.6472 & 0.7071 & -0.7071 \\
15 & 0.2369 & -0.8841 & 0.2585 & -0.9659 \\
\hline
\end{tabular}


TABLE III. Boundary data for ellipse, $P_{0}=10^{4}$ p.s.i.

Semiaxes: $a=200$ in., $b=100$ in.;

Poisson's ratio $\nu=.20$,

Young's Modulus $E=10^{\circ}$ p.s.i.

Boundary $u_{i \mu}($ in. $)$

Numerical

Analytical

\begin{tabular}{c|c|c|c|c}
\hline Node & $u_{1 \mu}$ & $u_{2 \mu}$ & $u_{1 \mu}$ & $u_{2 \mu}$ \\
\hline 19 & 0 & 0.3541 & 0 & 0.3600 \\
20 & 0.1434 & 0.3484 & 0.1454 & 0.3526 \\
21 & 0.2849 & 0.3232 & 0.2887 & 0.3298 \\
22 & 0.4226 & 0.2824 & 0.4277 & 0.2896 \\
23 & 0.5507 & 0.2203 & 0.5587 & 0.2271 \\
24 & 0.6554 & 0.1280 & 0.6689 & 0.1332 \\
1 & 0.7008 & 0 & 0.7200 & 0 \\
\hline
\end{tabular}

A simple traction problem for this domain is posed by loading the two short sides with a uniform normal tensile traction $t_{1}$. Tractions everywhere else are zero. Although this problem is most elementary, the character of the boundary and discontinuity of the prescribed traction are features not previously encountered. Boundary displacement components are calculated and data for the first quadrant are given in Table IV.

As a check on field quantities, displacement components at points along several radii of the circular domain with bcundary traction $N=P_{0} \sin ^{2} \phi$ were calculated. Agreement with analytically computed field values is comparable or better than that obtained for the given boundary quantities. Also, a check on the stresses at the same points using a simple finite difference procedure was obtained with rati $\wedge$ surprising accuracy considering there are two levels of approximation involved to ot .in stresses in this manner. However, the finite difference procedure need not be used since once the boundary quantities are determined, Eq. (2.5) can be differentiated directly to yield an expression for the stresses. Thus, one level of approximation is eliminated. Since all such differentiations are with respect to the field point $p$, only the kernel functions are effected.

TABLE IV. Boundary data for rectangular domain, $t_{1}=10^{4}$ p.s.i.

Dimensions: 360 in. $\times 200$ in.;

Poisson's ratio v $=.20$, Young's Modulus $E=10^{\circ}$ p.s.i. Boundary $u_{i \mu}($ in.)

Numerical

Analytical

\begin{tabular}{c|c|c|c|c}
\hline Node & $u_{1 \mu}$ & $u_{2 \mu}$ & $u_{1 \mu}$ & $u_{2 \mu}$ \\
\hline 22 & 0 & $\cdots 0.2208$ & 0 & -0.2000 \\
23 & 0.3991 & -0.2213 & 0.4000 & -0.2000 \\
24 & 0.7976 & -0.2227 & 0.8000 & -0.2000 \\
25 & 1.1941 & -0.2249 & 1.2000 & -0.2000 \\
26 & 1.5817 & -0.2250 & 1.6000 & -0.2000 \\
27 & 1.7909 & -0.1495 & 1.8000 & -0.1600 \\
28 & 1.8048 & -0.0773 & 1.8000 & -0.0800 \\
1 & 1.8065 & 0 & 1.8000 & 0 \\
\hline
\end{tabular}


Again, if the known boundary quantities are assumed constant over the boundary intervals, equations for the stresses of the form of Eq. (3.2) are immediately obtained in which the appropriate integrals may be evaluated in a fashion similar to that outlined in Sec. 3.

Details on the direct stress calculations and more extensive stringent tests of the proposed method for calculating associated boundary quantities are currently under investigation. Questions of effectiveness still remain.

First, an obvious test for a given problem is to start with $n$ small and examine the computed data as $n$ increases. The reasonably successful results presented for so few nodal points surely are in some measure due to the simple geometry of the domains and smooth nature of the boundary data. The numbers $n$ used were chosen for convenience; the associated amount of data along with the computer program fit easily on the core of the (IBM 7094) computer. Attention in this first trial of the procedure was focused on testing the approximations for the three types of boundary value problems for at least the variety of regions chosen, rather than on optimum programming, convergence, or error analysis. The extent to which $n$ may be increased, above which ill-conditioning may result in a given problem is as yet unknown. Also, significant increases in $n$ require additional computer storage or at least some attention to programming optimization. Tape storage, at present, increases computer time, but no problem presented took more than three minutes on the computer.

Except for the circular ring, nodal spacing is equal around the boundary. This would seem desirable for optimum conditioning of the equations if the remarks of Symm [10] are applicable here. To what extent unequal intervals may be used to perhaps handle rapidly varying boundary data more effectively, also remains to be investigated.

Any specific discussion of the limited amount of data presented regarding the above matters would, of course be inconclusive. The ss 'utions presented serve, however, to solidly indicate the scope of the method. Difficulties ncountered in more comprehensive problems will be of a tactical nature to be overcome by a refinement in numerical procedures.

Inherent in the boundary formula procedure is an effective reduction in dimension in which all approximations take place only on the boundary of the domain. Still, a relatively high capacity high speed computer is required for the method to be generally practicable. The wide availability of these is responsible for the fact that integral equations need no longer be useful only for theoretical investigations. Indeed, it is envisaged that solutions to complicated boundary value problems, tractable perhaps by no other means, may be expediently found by integral equation methods.

Acknowledgment. Thanks are due to Professors M. Stippes, M. Stern, and M. A. Jaswon, for valuable discussions and helpful comments on the original draft, and to Mr. C. C. Chang for programming and gathering numerical data.

\section{REFERENCES}

1. E. Betti, II Nuovo Cimento 6-10, (1872)

2. C. Somigliana, Il Nuovo Cimento 17-20, (1885)

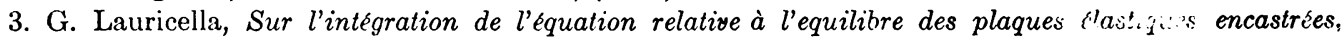
Acta Math. 32, (1909)

4. I. Fredholm, Solution d'un problème fondamental de la theorie de l'elasticité, Arch. Mat. .sit'onor... Fysik 2, (1905)

5. D. I. Sherman, Dokl. Akad. Nauk SSSR 27-28, (1940); 32, (1941) 
6. S. G. Mikhlin, Integral equations, Pergamon Press, London, 1957

7. N. I. Muskhelishvili, Some basic problems of the mathematical theory of elasticity, Noordhoff, Holland, 1953

8. M. A. Jaswon, Integral equation methods in potential theory. I, Proc. Roy Soc. Ser. A 273, (1963).

9. M. A. Jaswon and A. R. Ponter, An integral equation solution of the torsion problem, Proc. Roy. Soc. Ser. $A$ 273, (1963)

10. G. T. Symm, Integral equation methods in potential theory. II. Proc. Roy. Soc. Ser. A 275, (1963)

11. A. E. H. Love, $A$ treatise on the mathematical theory of elasticity, 4th ed., Dover, New York, 1944

12. N. I. Muskhelishvili, Singular integral equations, Noordhoff, Holland, 1953

13. W. D. MacMillan, The theory of the potential, Dover, New York, 1958 